\title{
EtNobotánica de Heliopsis lONGIPES (Asteraceae: Heliantheae)
}

\author{
V. Gabriela Cilia-López ${ }^{1,3}$, J. Rogelio Aguirre-Rivera², Juan Antonio Reyes-Agüero² \\ Y BERTHA I. JUÁREZ-FLORES ${ }^{2}$. \\ 'Posgrado en Ciencias Ambientales \\ ${ }^{2}$ Instituto de Investigación de Zonas Desérticas \\ Universidad Autónoma de San Luis Potosí Altair 200, Fracc. Del Llano C.P. 78377, San Luis Potosí, S. L. P., México \\ ${ }^{3}$ Autor para la correspondencia. Correo-e: pmpca_gcilia@yahoo.com.mx
}

\begin{abstract}
Resumen: Heliopsis longipes, conocido con el nombre común de chilcuague es una hierba perenne endémica de la sierra de Álvarez y sierra Gorda, región donde coinciden parte de los territorios de San Luis Potosí, Guanajuato y Querétaro, y de donde se conoce que su raíz tiene usos tradicionales. Los objetivos de este trabajo fueron registrar las formas actuales de uso tradicional del chilcuague y estimar la demanda actual de su raíz. Se realizaron entrevistas a recolectores, comerciantes y consumidores en los mercados tradicionales de San Luis Potosí, Guanajuato y Querétaro. La raíz de chilcuague es económica y culturalmente importante, tiene diversos usos tradicionales y su venta se practica todo el año en los tianguis de San Luis de la Paz, Dr. Mora y San José Iturbide, Guanajuato; Rioverde y San Ciro, San Luis Potosí; y en Jalpan, Querétaro. Como resultado de este trabajo se encontró una forma inédita del uso de chilcuague como desparasitante, adicionando las raíces a los alimentos. Asimismo, se encontró una relación directa entre la edad de los informantes y su conocimiento sobre la planta, y más aún, en las localidades donde se conocen más usos del chilcuague es en donde hay una mayor frecuencia de venta.
\end{abstract}

Palabras clave: chilcuague, desparasitante, Sierra Gorda, usos tradicionales.

\begin{abstract}
Heliopsis longipes known as chilcuague is a perennial herb endemic to Sierra de Álvarez and Sierra Gorda, where the States of San Luis Potosí, Guanajuato and Querétaro converge, and its root has traditional uses. However, there is a lack of studies that document the current traditional uses within its entire distribution area. The objectives of this research were to determine the traditional forms of use of chilcuague, and to determine the current demand of its root. Gathers, merchants, and consumers in traditional markets (tianguis) in San Luis Potosí, Guanajuato and Querétaro were interviewed. The root of chilcuague is economically and culturally important. It has diverse traditional uses and its sale is practiced all year-long at the traditional markets of San Luis de la Paz, Dr. Mora, and San José Iturbide, Guanajuato; Rioverde and San Ciro, San Luis Potosí; and Jalpan, Querétaro. Chilcuague is used against parasites by adding roots over food. Moreover, a correlation with age of informants and knowledge of the uses of the plant was found. A higher amount of dried roots of chilcuague were sold in places with more traditional uses.
\end{abstract}

Key words: chilcuague, parasites, traditional uses, Sierra Gorda

$\mathbf{L}^{2}$ a relación prolongada de los humanos con las plantas generó un conocimiento diverso sobre los recursos vegetales, lo cual explica que algunas plantas formen parte importante de la economía mundial. Sin embargo, generalmente las plantas silvestres económicamente importantes carecen de suficiente información acerca de su distribución, estado de conservación de su hábitat, estudios poblacionales, requerimientos ecológicos y de sus formas de uso tradicional (Gálvez y de Ita, 1992). Heliopsis longipes S.F. Blake es una hierba perenne endémica de la región conformada por porciones de la Sierra de Álvarez y la Sierra Gorda, donde coinciden parte de los Estados de San Luis Potosí, Guanajuato y Querétaro (figura 1) (Little, 1948 a, b). Los nombres comunes de esta planta son chilcuague, pelitre, raíz de oro y raíz azteca (Ramírez, 1902; Santamaría, 1959; Martínez, 1967).

Dentro del género, Heliopsis longipes es la especie con mayor importancia económica (Fisher, 1954); además de sus cualidades insecticidas y medicinales reconocidas, su raíz se usa como condimento en salsas, guisos y en bebidas alco- 


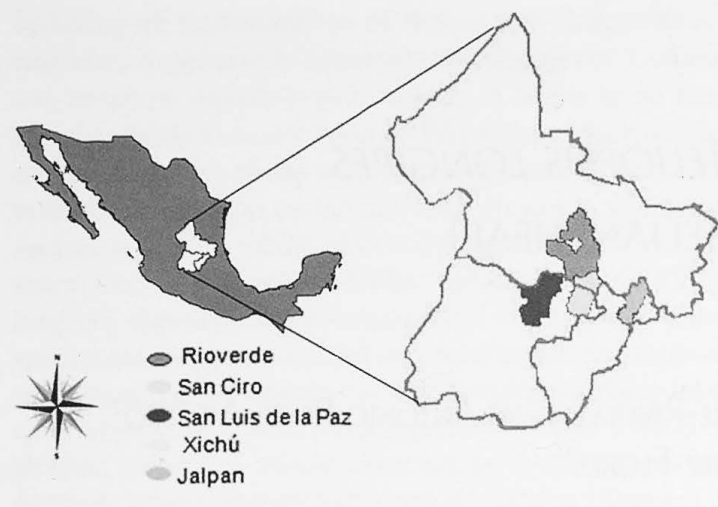

Figura 1. Distribución geográfica conocida de Heliopsis longipes.

hólicas (Martínez, 1967). En medicina tradicional se emplea para calmar dolores musculares y de muelas, así como desparasitante e insecticida (Little, 1948b; Martínez, 1967). El uso del chilcuague ha implicado la destrucción total de la planta por lo que las poblaciones de esta especie han disminuido, desapareciendo en algunas zonas (Little, 1948b; Molina-Torres y García-Chávez, 2001). Se han realizado estudios químicos (Jacobson, 1954; Jacobson 1955; Domínguez et al., 1958; Molina-Torres et al., 1995; Molina-Torres et al., 1996; García-Chávez et al., 2004) y farmacológicos (Johns et al., 1982; Romero et al., 1989; Gutiérrez-Lugo et al., 1996; Molina-Torres et al., 1999; Ramírez-Chávez et al., 2000) con la raíz de H. longipes. Sin embargo, se carece de estudios recientes que documenten el estado actual de los usos tradicionales y la importancia económica de esta especie en su área de distribución natural. Por lo anterior, los objetivos del presente trabajo fueron registrar las formas actuales de uso tradicional de $H$. longipes y conocer la demanda actual de la raíz de $H$. longipes en la región donde se distribuye.

\section{Materiales y métodos}

Registro de la información etnobotánica. Para recabar el conocimiento tradicional sobre $H$. longipes, se elaboraron cuestionarios dirigidos a las personas involucradas en el conocimiento y uso del chilcuague, como recolectores, comerciantes y usuarios (Montes y Aguirre, 1994). Las entrevistas se realizaron el día de tianguis en nueve mercados tradicionales de los Estados de San Luis Potosí, Guanajuato y Querétaro, situados dentro y en los alrededores del área de distribución natural conocida de H. longipes. Las entrevistas fueron abiertas, pero basadas en los cuestionarios elaborados para los diferentes grupos, lo cual facilitó el intercambio de información.

Los comerciantes entrevistados $(n=23)$ fueron personas con experiencia en la venta de chilcuague. Los recolectores $(n=2)$ fueron campesinos dedicados a la recolección de esta planta para su venta; estos recolectores se seleccionaron con base en lo propuesto por Hernández y Ramos (1977); es decir, fueron los recolectores con mayor experiencia en la recolecta de esta raíz. Los usuarios entrevistados $(n=15$ en cada uno de los nueve mercados) fueron personas que adquirían el chilcuague y se comprobaba que tenían conocimiento de los usos o propiedades de esta planta. Las entrevistas se realizaron de febrero a noviembre de 2004 , durante los días de tianguis en los diferentes mercados explorados. Se registraron los usos y el número de menciones de los mismos para calcular la frecuencia de uso de raíz de chilcuague en la región.

Demanda actual de raíz de chilcuague en la región. Con base en los cuestionarios dirigidos a los recolectores y comerciantes, se registraron las unidades de venta de chilcuague (manojos), el peso y precio unitario, los puntos de. venta, la temporada y frecuencia de venta en cada uno de los mercados visitados. Con los datos obtenidos se calcularon las estimaciones para obtener el volumen anual, el precio por kilogramo y el valor anual, de manera similar al Valor de Importancia Etnobotánica usado por Vargas et al. (1994), mediante las siguientes ecuaciones:

Volumen

$$
\text { anual }=\frac{\text { cantidad de manojos vendidos en un día }}{\frac{\text { de tianguis } \times \text { peso del manojo }(\mathrm{g}) \times 4 \times 12}{1000 \mathrm{~g}}}
$$

Donde:

4 = número de días de tianguis por mes

$12=$ meses del año

$\mathrm{g}=$ gramos

$$
\text { Precio por kilogramo }=\frac{\text { peso del manojo }(\mathrm{g})}{\frac{\mathrm{x} \text { precio de venta }}{1000 \mathrm{~g}}}
$$

Valor anual $=$ precio por kilogramo $\mathrm{x}$ volumen anual

\section{Resultados}

Nomenclatura, conocimiento y usos tradicionales. El nombre común predominante para Heliopsis longipes en la región es chilcuague, con la variante de chilcuán; otros nombres a veces mencionados fueron pelitre y raíz de oro. De acuerdo con las personas entrevistadas, en ninguno de los mercados visitados se conoce otra planta con los mismos nombres. De $H$. longipes sólo se usa la raíz, la cual se emplea para diferentes propósitos (figura 2). En Guanajuato, San Luis Potosí y Querétaro el uso más frecuente es como condimento en salsas y diversos guisos; para este propósito se usan de cinco a siete raíces de chilcuague molidas.

Según los informantes, el gusto por la raíz de chilcuague se debe a su sabor pungente y a la insensibilización que produce, por lo que se le combina con chile o como substituto 


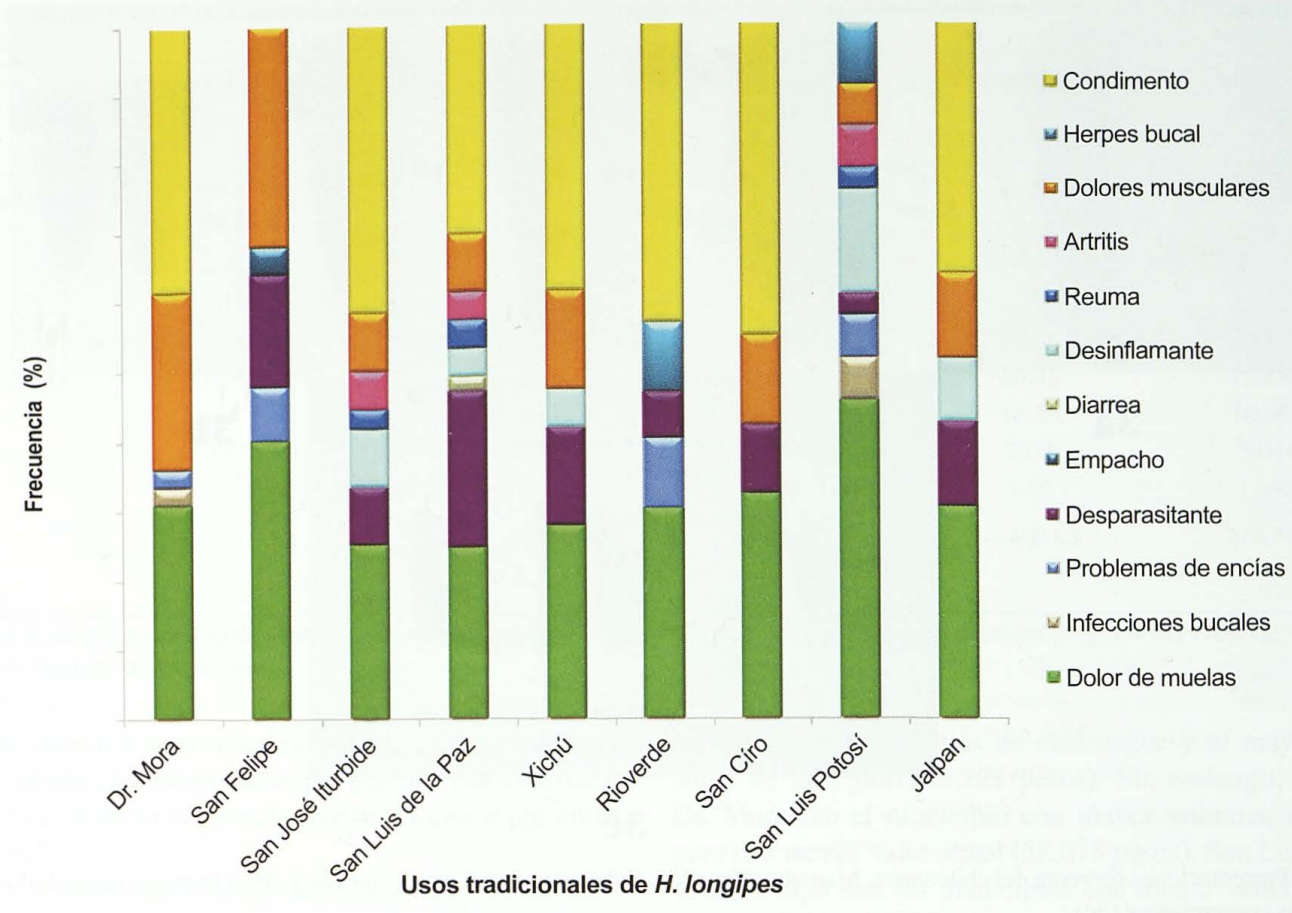

Figura 2. Formas de uso tradicional declaradas por compradores de raíz de Heliopsis longipes en los mercados de Guanajuato, San Luis Potosí y Querétaro donde se comercializa.

del mismo. En medicina tradicional se usa para calmar dolores de muelas, para lo cual se coloca y retiene un trocito de raíz sobre el diente afectado; para calmar dolores musculares se le emplea macerada en alcohol; como desparasitante, en ayunas se mastica un trozo de raíz, o bien, se consume cotidianamente en los alimentos (figura 2).

Prácticas de recolección, venta y consumo de la raíz de chilcuague. De los dos recolectores seleccionados, uno opera en el municipio de Rioverde, S.L.P. y el otro en San Luis de la Paz, Gto. Ambos viven de la recolecta de chilcuague y de otras especies de plantas silvestres desde hace más de 15 años, actividad que realizan durante todo el año. El recolector de San Luis de la Paz vende los manojos de chilcuague directamente a los vendedores del mercado de este municipio; el recolector de Rioverde los vende a un intermediario o acopiador, quien posteriormente los revende a los comerciantes establecidos en el mercado de este municipio. Ambos recolectores dedican uno o dos días del mes a la recolecta de raíz de chilcuague; por jornada extraen las raíces de 50 a 100 plantas para formar de 20 a 30 manojos (manojos primarios, figura $3 \mathrm{a}$ y $3 \mathrm{~b}$ ).

Cada manojo lo integran con las raíces de cinco a siete plantas, y un manojo puede tener de 120 a $200 \mathrm{~g}$ de peso; es decir por jornada, cada recolector obtiene entre 2400 y 6000 g de raíz de chilcuague. Cabe señalar que los vástagos de las plantas extraídas son desechados. Ninguno de los informantes cultiva el chilcuague; todas las raíces las obtienen de poblaciones silvestres. Ambos reconocen que la abundancia de esta planta ha disminuido en los últimos 20 años, pues antes era más fácil y común encontrarla en los parajes de los bosques de encino y encino-pino de la Sierra de Álvarez y Sierra Gorda, en los Municipios de Rioverde y de San Luis de la Paz de donde son originarios.

Venta. Existen dos tipos de comerciantes que se dedican a la venta del chilcuague, los establecidos y los informales. Los comerciantes establecidos tienen un local determinado dentro del mercado municipal, por lo general son locales especializados en venta de plantas medicinales o herbolarias; aunque también el chilcuague se vende en locales de frutas y verduras. Los comerciantes informales realizan su venta fuera del edificio del mercado, en locales improvisados y sólo durante el día de tianguis.

Con el propósito de explorar otros municipios cercanos al área de distribución conocida de H. longipes, se visitaron los mercados de San Felipe y Dolores Hidalgo, municipios próximos a la Sierra de Guanajuato. En Dolores Hidalgo se visitaron las tres herbolarias existentes, de las cuales sólo una vende chilcuague; las otras dos dejaron de venderlo de- 


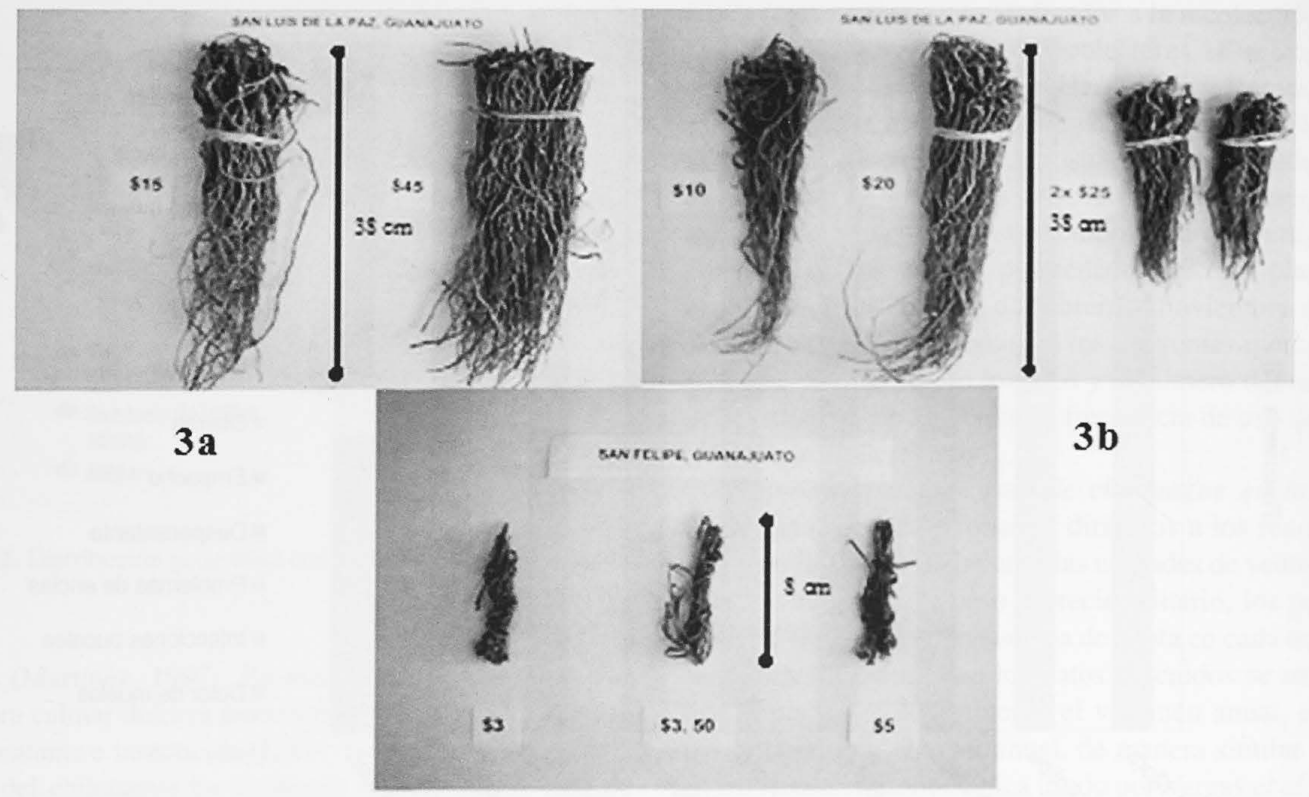

\section{$3 c$}

Figura 3. Presentaciones de venta del chilcuague. Manojos primarios, elaborados por el recolector (3a y 3b) y manojos secundarios, elaborados por los comerciantes (3c).

bido al incremento en el precio por manojo que ocasionó disminución en su consumo; la raíz que se vende en este municipio se compra en San Luis de la Paz. En San Felipe se encontraron cuatro herbolarias que venden chilcuague, la raíz que se ofrece en este municipio se compra en la ciudad de San Luis Potosí.

El comercio y la unidad de venta de la raíz de chilcuague en todos los municipios visitados son en manojos (figura 3). Los manojos que forma el recolector (manojos primarios figura 3a y 3b) son entregados a los comerciantes en los mercados y tianguis de Dr. Mora, San Luis de la Paz y San José Iturbide (Gto.), Rioverde y San Ciro (S.L.P.) y en Jalpan (Qro.). El peso de los manojos que se venden en estos municipios varía generalmente entre 30 y $200 \mathrm{~g}$. Los comerciantes elaboran a partir de los manojos primarios los manojos secundarios (figura 3a), los cuales son mucho más pequeños, pues sólo pesan entre 2 y $4 \mathrm{~g}$ y son los que se ofrecen en herbolarias de la ciudad de San Luis Potosí y de San Felipe, Gto. Los ejemplares corroborativos de $H$. longipes se encuentran depositados en el herbario SLPM (Cilia 113 42505, Cilia 114 42502, Cilia 116 42501, Cilia 117 42504).

La edad de los comerciantes entrevistados se encontró entre 21 y 77 años; la antigüedad que tenían vendiendo chilcuague resultó muy variable, pues para algunos fue de sólo un año, mientras que para otros fue de 30 y hasta 50 años, por lo que el conocimiento sobre los usos tradicionales entre ellos fue muy heterogéneo. Los más jóvenes desconocían el lugar de procedencia de la especie, así como algunas de sus formas de uso; los de más de 35 años proporcionaron información detallada de los usos, preparaciones, distribución, demanda, etc. Ninguno de los comerciantes entrevistados en todos los mercados visitados resultó ser productor o recolector de chilcuague, ya que todos obtienen la raíz directamente de los recolectores o de intermediarios.

Consumo. Las personas entrevistadas con más de 30 años de edad conocen bien los usos tradicionales del chilcuague y la mayoría suele utilizarlo; las personas más jóvenes (de 17 a 30 años) conocen poco sobre la planta, saben de algunos de sus usos, pero no la consumen. Los consumidores de la ciudad de San Luis Potosí y de San Felipe sólo conocen los usos medicinales del chilcuague y en la actualidad lo utilizan esporádicamente. En Rioverde y San Ciro, en San Luis de la Paz, Xichú, Dr. Mora y San José Iturbide, y en Jalpan, la raíz de chilcuague se consume principalmente como condimento y para calmar el dolor de muelas (figura 2). En San Luis de la Paz, los consumidores mencionaron que la ingestión cotidiana de la raíz en la comida evita o elimina los parásitos. Sólo en este municipio se registró la elaboración de "burritos con chilcuague", que son tortas de masa de maíz rellenas con frijoles o nopales condimentados con raíces de chilcuague; estos burritos son tradicionales y aparentemente exclusivos de este municipio. En Dolores Hidalgo, los consumidores compraban el chilcuague para calmar 


\section{ETNOBOTÁNICA DE HELIOPSIS}

Cuadro 1. Cantidad y valor de la venta anual (2004) de raíz de chilcuague en mercados de San Luis Potosí, Guanajuato y Querétaro donde se comercializa.

\begin{tabular}{|c|c|c|c|c|c|}
\hline Localidad & Puntos de venta & $\begin{array}{c}\text { Manojos } \\
\text { vendidos por } \\
\text { día de tianguis }\end{array}$ & $\begin{array}{l}\text { Precio medio } \\
{\text { (pesos } / \mathrm{kg})^{\mathrm{a}}}^{\text {a }}\end{array}$ & $\begin{array}{l}\text { Volumen anual } \\
\qquad(\mathrm{kg})^{\mathrm{b}}\end{array}$ & $\begin{array}{c}\text { Valor anual } \\
\text { (pesos) }^{c}\end{array}$ \\
\hline San Luis de la Paz & 10 & 86 & $1,049.82$ & 86.62 & 85,121 \\
\hline Dr. Mora & 10 & 63 & 484.60 & 136.50 & 58,078 \\
\hline Rioverde & 9 & 67 & 961.82 & 75.71 & 55,874 \\
\hline Jalpan & 6 & 22 & 603.86 & 43.72 & 26,400 \\
\hline Xichú & 4 & 22 & 233.84 & 84.58 & 20,400 \\
\hline San José Iturbide & 5 & 26 & 409.84 & 30.45 & 12,480 \\
\hline San Ciro & 4 & 15 & 588.23 & 18.36 & 10,000 \\
\hline San Luis Potosí & 10 & 20 & $1,179.45$ & 3.62 & 5,044 \\
\hline San Felipe & 4 & 8 & $1,427.92$ & 1.07 & 1,393 \\
\hline Total & 62 & 329 & --- & ---480.63 & 274,790 \\
\hline Promedio & --- & ---- & 771.04 & & ---- \\
\hline
\end{tabular}

${ }^{\mathrm{a}}=$ peso del manojo $(\mathrm{g}) \times$ precio de venta $/ 1000 \mathrm{~g} ;{ }^{\mathrm{b}}=$ manojos vendidos en un día de tianguis $\times$ peso del manojo $(\mathrm{g}) \times 4 \times 12 / 1000 \mathrm{~g} ;{ }^{\mathrm{c}}=\mathrm{precio}$ por $\mathrm{kg} \times$ volumen anual; $\mathrm{g}=$ gramos

el dolor de muelas y como condimento; sin embargo, dejaron de usarlo debido a los precios altos por manojo. Los usos tradicionales del chilcuague registrados por municipio se presentan en la figura 2.

Demanda actual de raíz de chilcuague. La raíz de chilcuague se vende durante todo el año en los mercados de San Luis de la Paz, Dr. Mora y San José Iturbide, Gto.; en Rioverde y San Ciro, S.L.P.; y en Jalpan, Qro., localidades cercanas al área de distribución conocida de esta especie. Las personas entrevistadas en cada uno de los municipios mencionados reconocen que hasta hace unos 10 años se vendía en mayor cantidad y era más barato. En la ciudad de San Luis Potosí y en el municipio de San Felipe la demanda de raíz de chilcuague es de uno a dos manojos al mes en cada herbolaria registrada, pues poca gente conoce sus usos; en Jalpan cada comerciante vende de dos a tres manojos por día de tianguis; en Rioverde, San Ciro, Xichú y San José Iturbide se venden de tres a cinco manojos por día de tianguis en cada punto de venta registrado; en Xichú cada puesto vende de cuatro a cinco manojos por día de tianguis, y en Dr. Mora de cinco a seis manojos. San Luis de la Paz fue la localidad con mayor demanda de chilcuague con cinco a siete manojos de chilcuague vendidos por día de tianguis en cada punto de venta registrado. Es importante mencionar que los datos presentados corresponden a un día de tianguis por semana, ya que en las herbolarias permanentes se vende la raíz de chilcuague todos los días del año. Los puntos de venta registrados en cada municipio visitado se presentan en el cuadro 1.

La estimación de la cantidad y valor de la venta anual de raíz de chilcuague en la región se muestra en el cuadro 1. Para el 2004 en la región se vendieron $480.63 \mathrm{~kg}$ con un valor de 274,790 pesos; sin embargo, cada municipio tuvo sus particularidades. San Luis de la Paz es el municipio con mayor venta de manojos de chilcuague y el mayor valor anual de la región $(85,121$ pesos). Sin embargo, aunque Dr. Mora fue el municipio con mayor volumen ofrecido, pero con menor valor anual (58,078 pesos). San Luis Potosí y San Felipe son los municipios con menor venta de chilcuague (3.62 y $1.07 \mathrm{~kg}$, respectivamente); sin embargo, su precio por kilogramo fue el más elevado de la región.

\section{Discusión}

El nombre chilcuague se deriva de chilmecatl, palabra compuesta de origen náhuatl, conformada por chilli o chili que significa picante o picoso, y mecatl que significa cordel; chilmecatl hace referencia a las raíces filiformes y de sabor pungente que caracterizan a H. longipes (Martínez, 1967). El nombre pelitre se relaciona con la pellitorina, amida descubierta en Anacyclus pyrethrum DC. (Asteraceae) especie nativa del norte de África, cuya raíz se emplea en medicina tradicional. Al igual que la raíz de $H$. longipes, la de Anacyclus pyrethrum es pungente y estimula la actividad salival (Gulland y Hopton, 1930). Por estas coincidencias, en México se nombró a $H$. longipes como pelitre del país o falso pelitre (Ramírez, 1902; Little, 1948a). De este modo, pelitre es nombre común de algunas asteráceas como $A$. pyrethrum, Acmella repens (Walter) Rich, Erigeron longipes y Spilanthes ocymifolia A.H. Moore, las cuales tienen en común raíces pungentes con propiedades medicinales (Gulland y Hopton, 1930; Little, 1948a; Santamaría, 1959). Por lo tanto, los nombres chilcuague y pelitre se deben al sabor pungente característico de las raíces de $H$. longipes.

Los usos tradicionales de la raíz de chilcuague registrados en la región (condimento y para calmar dolores de muelas y musculares) coinciden con lo mencionado por Little (1948a, 1948b), Martínez (1967) y Salazar (1999); 
sin embargo, dichos autores no mencionan el uso de esta planta como desparasitante de uso común incorporado en la comida. Los usos conocidos han persistido en la región de estudio por más de 60 años desde que fueron publicados (Little, 1948 a, b); además, en todos los mercados visitados, se desconoce otra planta que presente las mismas propiedades o características que la raíz de $H$. longipes. Las formas de uso inéditas registradas en el presente trabajo, y la confirmación de los usos consignados en los antecedentes principales, indican que el chilcuague continúa siendo una especie culturalmente importante en su área de distribución natural.

De la raíz de $H$. longipes se aisló una alcamida, la $\mathrm{N}$ isobutil-2, 6, 8-decatrienoamida $\left(\mathrm{C}_{14} \mathrm{H}_{23} \mathrm{NO}\right)$; a este compuesto se le llamó afinina y se le atribuyó la propiedad insecticida (Acree et al., 1945). En la raíz de $H$. longipes se ha registrado la presencia de azúcares, flavonoides y terpenos (Salazar, 1999), y en las hojas la presencia de esteroles, terpenos y flavonoides (Cárdenas, 2005). Estudios con plantas silvestres realizados por el Departamento de Agricultura de los Estados Unidos (Roak, 1947; Little, 1948a) demostraron que extractos de la raíz de $H$. longipes presentan el mismo efecto tóxico y paralizante sobre las moscas que el piretro (Chrysanthemum cinerariaefolium Vis). Se han realizado diversos estudios para evaluar las propiedades biocidas de la raíz de H. longipes; de este modo, Jacobson et al. (1947) registraron efecto insecticida de la afinina sobre la mosca doméstica, y Domínguez et al. (1958) lo observaron sobre el gorgojo del frijol (Acanthoseelides octectus Say). También se ha verificado que la afinina presenta acción molusquicida (Johns et al., 1982) y fungicida (Ramírez-Chávez et al., 2000), y se ha demostrado que extractos de su raíz tienen acción anti-infecciosa sobre diversas bacterias y hongos (Gutiérrez-Lugo et al., 1996). Juárez et al. (2001) evaluaron el efecto insecticida de hojas, flores y raíces de $H$. longipes, y encontraron que únicamente la raíz presenta acción insecticida, lo cual explica el uso tradicional de sólo la raíz de esta planta. Sin embargo, Cárdenas (2005) sí encontró efecto fungicida del follaje. La presencia de afinina en la raíz de $H$. longipes y su propiedad biocida, parece justificar su uso tradicional como desparasitante intestinal cotidiano por los pobladores de la región geográfica de esta especie.

La edad de los informantes influyó en la cantidad y calidad de la información etnobotánica proporcionada; respecto a los comerciantes entrevistados, los más jóvenes desconocen el lugar de procedencia de la especie y sus formas de uso, mientras que los informantes mayores que 35 años proporcionaron mejor información acerca de los usos, formas de preparación, distribución y demanda de la raíz de chilcuague. La misma situación se presentó con los usuarios o consumidores, ya que las personas entrevistadas con más que 30 años de edad conocen bien los usos tradicionales del chilcuague y en su mayoría sigue consumiéndolo; por su parte, los informantes con menos que 30 años saben poco acerca de los usos del chilcuague y la mayoría no lo consumen. Esta relación directa entre la edad del informante y su conocimiento etnobotánico coincide con lo documentado por Estrada et al. (2001) en una región boscosa del estado de México.

La frecuencia de venta de chilcuague está directamente relacionada con el conocimiento sobre los usos tradicionales del chilcuague; así, en los mercados de San Luis de la Paz, Dr. Mora, Rioverde y Jalpan se venden en promedio de cuatro a cinco manojos en cada punto de venta durante los días de tianguis y es en estos municipios donde se vende el mayor volumen y se alcanza el mayor valor anual de venta en la región (cuadro 1), y lo contrario sucede en San Luis Potosí y San Felipe, donde la venta es esporádica, de uno a dos manojos al mes, ya que poca gente conoce sus usos. Los precios más altos por manojo en la región se registraron en Doctor Mora y San Luis de la Paz (30 y 50 pesos, respectivamente), y los más bajos en la ciudad de San Luis Potosí y en San Felipe (3.00 pesos) aunque con tamaños muy distintos. Estos datos concuerdan con lo registrado por Salazar (1999), pues para la ciudad de San Luis Potosí el manojito de raíz se vendía en 0.50 pesos y en San Luis de la Paz en 18 pesos en ese año. En cuanto al valor de la raíz por kilogramo, los precios son mayores en los mercados con mayor intermediación (Rioverde, San Luis de la Paz, San Luis Potosí y San Felipe).

El conocimiento amplio sobre las formas de uso tradicional de la raíz de chilcuague está directamente relacionado con la mayor frecuencia de venta, y esto ocurre en San Luis de la Paz, Doctor Mora y Rioverde, municipios situados dentro del área más probable de distribución natural de $H$. longipes. La pobreza del conocimiento sobre los usos tradicionales y la procedencia foránea de la raíz de chilcuague ofrecida en los mercados de San Felipe y Dolores Hidalgo más la ausencia de referencias escritas y de recolectas botánicas, son indicadores que hacen poco probable que la Sierra de Guanajuato sea parte del área de distribución natural del chilcuague; sin embargo es necesaria la exploración florística de esta zona para corroborar su ausencia en esta Sierra. Así, Heliopsis longipes es una especie silvestre económica y culturalmente importante, con diversos usos tradicionales en la región de la que es endémica. La venta de la raíz de chilcuague se practica durante todo el año en los tianguis de San Luis de la Paz, Dr. Mora y San José Iturbide en Guanajuato; en Rioverde y San Ciro en San Luis Potosí y en Jalpan, Querétaro. Es probable que la disponibilidad de este recurso se esté reduciendo por su forma de aprovechamiento actual destructiva y porque su utilización ha persistido en las localidades asociadas a su área de distribución. Es importante realizar estudios poblacionales y ecológicos para conocer el estado actual de las poblaciones silvestres, así como estudios sobre la propagación de H. longipes, para disminuir la presión del aprovechamiento actual en las poblaciones silvestres. 


\section{Literatura citada}

Acree F. Jr., Jacobson M. y Haller H.L. 1945. The structure of affinin, the insecticidal amide from Erigeron affinis DC. Journal of Organic Chemistry. 10: 449-451.

Cárdenas O.N.C. 2005. Actividad antifúngica de cuatro especies de plantas sobre Aspergillus flavus Link. Tesis doctoral. División de Ciencias Biológicas y de la Salud. Universidad Autónoma Metropolitana, Unidad Xochimilco. México D.F. 131 pp.

Domínguez J.A., Leal D.G. y Viñales D.M.A. 1958. Síntesis de Nisopropil y $\mathrm{N}$-isobutilamida de algunos ácidos y comparación de su acción insecticida con la afinina. Ciencia. 17: 213-216.

Estrada M.E., Aguirre R.J.R. y Sánchez R.L. 2001. Tecnología tradicional y conocimiento etnobotánico forestal en Santa Isabel Chalma, Amecameca, México. Revista de Geografía Agricola. 32: 43-74

Fisher T.R. 1954. Taxonomy of the genus Heliopsis (Compositae). Tesis doctoral. Departamento de Botánica. Indiana University. Bloomington, Indiana. USA. 156 pp.

Gálvez C.M.C y de Ita C.M. 1992. Análisis etnobotánico de tres mercados regionales del centro del Estado de Veracruz. Tesis profesional. Universidad Veracruzana. Córdoba, Veracruz. 162 pp.

García-Chávez A., Ramírez C.E. y Molina-Torres J. 2004. El género Heliopsis (Heliantheae: Astercaeae) en México y las alcamidas presentes en sus raíces. Acta Botánica Mexicana. 69:115-131.

Gulland M. J y Hopton G.U. 1930. Pellitorine the pungent principle of Anacyclus pyretrhum. Journal of the Chemical Society. 132: 6-11.

Gutiérrez-Lugo M.T., Barrientos-Benítez T., Luna B., RamírezGama R.M., Bye R., Linares E. y Mata R. 1996. Antimicrobial and cytotoxic activities of some crude drug extracts from Mexican medicinal plants. Phytomedicine. 2: 341-347.

Hernández X. y Ramos R.A. 1977. Metodología para el estudio de agroecosistemas con persistencia de tecnología agrícola tradicional. Publicación especial de la Revista de Geografía Agrícola Xolocotzia. 1: 189-195.

Jacobson M. 1954. Constituents of Heliopsis species. III. Cis-trans isomerism in affinin. Journal of the American Chemical Society. 76: 4606-4608.

Jacobson M. 1955. Constituents of Heliopsis species. IV. The total synthesis of trans-affinin. Journal of the American Chemical Society. 77: 2461-2463.

Jacobson M., Acree F. y Haller H.L. 1947. Correction of the source of "affinin" (N-Isobutyl-2,6,8-decatrienoamide). The Journal of Organic Chemistry 12: 731-732.

Johns T., Graham K. y Towers G.H.N. 1982. Molluscicidal activity of affinin and other isobutylamides from the Asteraceae. Phytochemistry. 21: 2737-2738.

Juárez F.B.I., Jasso P.Y., Castillo C.R. y Aguirre R.J.R. 2001. Actividad del chilcuague Heliopsis longipes (Asteraceae) sobre el gorgojo del maíz Sitophilus zeamais (Coleoptera: Curculioni- dae). En: Rodríguez H.C. Ed. Memorias del II Simposio Internacional y VII Nacional sobre Sustancias Vegetales y Minerales en el Combate de Plagas. pp. 43-48. Querétaro, Querétaro. México.

Little E.L. 1948a. Heliopsis longipes, a Mexican insecticidal plant species. Journal of the Washington Academy of Sciences. 38:269-274.

Little E.L. 1948b. El chilcuague. Boletín de la Sociedad Botánica de México. 7: 23-27.

Martínez M. 1967. Las plantas medicinales de México. Botas. México.

Molina-Torres J., Salgado-Garciglia R., Ramírez-Chávez E., Del Río R.E. 1995. Presence of the bornyl ester of deca-2E,6Z,8Etrienoic acid in Heliopsis longipes roots. Journal of Natural Products. 58: 1590-1591.

Molina-Torres J., Salgado-Garciglia R., Ramírez-Chávez E. y Del Río R.E. 1996. Purely olefinic alkamides in Heliopsis longipes and Acmella (Spilanthes) oppositifolia. Biochemical Systematics and Ecology. 24: 43-47.

Molina-Torres J., García-Chávez A. y Ramírez-Chávez. E. 1999. Antimicrobial properties of alkamides present in flavouring plants traditionally used in Mesoamerica: affinin and capsaicin. Journal of Ethnopharmacology. 64: 241-248.

Molina-Torres J. y García-Chávez A. 2001. Alcamidas en plantas; distribución e importancia. Avance y Perspectiva. 20: 337-387.

Montes H.S. y Aguirre R.J.R. 1994. Etnobotánica del tomate mexicano (Physalis philadelphica Lam.). Revista de Geografía Agrícola. 20: 163-172.

Ramírez Chávez E., Lucas-Valdez L., Virgen-Calleros G. y Molina-Torres J. 2000. Actividad fungicida de la afinina y del extracto crudo de raíces de $H$. longipes en dos especies de Sclerotium. Agrociencia. 34: 207-215.

Ramírez J. 1902. Sinonimia vulgar y científica de las plantas mexicanas. Oficina Tipográfica de la Secretaría de Fomento. México.

Roark, R.C. 1947. Some promising insecticidal plants. Economic Botany. 1: 437-445.

Romero R. C.M., Del Castillo R.A.R, Martínez M.A.C. y Calderón F.C.J. 1989. Estudios preliminares de los efectos antibacterianos, insecticidas y toxicológicos de la raíz del chilcuán (Heliopsis longipes). Veterinaria Mexicana. 20: 151-156.

Salazar N.N.G. 1999. Farmacoetnología del chilcuague Heliopsis longipes (A. Gray) Blake. Tesis profesional. Facultad de Ciencias Químicas. Universidad Autónoma de San Luis Potosí, San Luis Potosí, México. 72 pp.

Santamaría J.F. 1959. Diccionario de mejicanismos. Porrúa. México.

Vargas N.A.A., Gálvez C.M.C., Aguirre R.J.R. y De Ita C.M. 1994. Métodos cuantitativos para el estudio etnobotánico de mercados regionales o tianguis. En: Cuevas S.J.A., Estrada L.E. y Cedillo P.E. Eds. Memorias del Primer Simposium Internacional sobre Etnobotánica en Mesoamérica. pp. 53-58. Universidad Autónoma Chapingo. México. 